\title{
Preclinical Safety Pharmacology Studies of Taiwanofungus camphoratus Extract
}

\section{Lin JY and Chiu E*}

Level Biotechnology Inc. CRO-Preclinical Testing Center, Taiwan

*Corresponding author: Emerson Chiu, Level Biotechnology Inc. CRO-Preclinical Testing Center, No. 80, Lane 169, Kangning Street, Hsi-Chih District, New Taipei City 221, Taiwan ROC, Tel: 886-02-2695-9935; Fax: 886-02-2692-1444; Email:

\section{Research article}

Volume 4 Issue 1

Received Date: February 12, 2019

Published Date: February 23, 2019

DOI: $10.23880 /$ act-16000146 emerson@mail.level.com.tw

\section{Abstract}

Taiwanofungus camphoratus is a unique mushroom that only grows in Taiwan. It has been used as fold medicine for a long history. Recent studies have demonstrated T. camphoratus possessed multiple pharmacological effects including anti-cancer, hepatoprotective and immunomodulatory effects. T. camphoratus extract was composed of extracts from cut$\log$ cultivated fruiting body and solid-state culture of T. camphoratus. This article presents the testing results of T. camphoratus extract in in vitro hERG assay and in vivo safety pharmacology studies on central nervous, respiratory and cardiovascular systems.

\section{Results}

1) The hERG transfected HEK293 cells were treated with T. camphoratus extract at concentrations of 5,10 and $25 \mu \mathrm{g} / \mathrm{mL}$ showed no significant effect on hERG current. 2) T. camphoratus extract was found to have no significant effects on central nervous and respiratory systems of male rats and female rats at oral doses up to $3400 \mathrm{mg} / \mathrm{kg}$ and $1700 \mathrm{mg} / \mathrm{kg}$, respectively. 3) Beagle dogs received T. camphoratus extract orally up to the dosage of $1000 \mathrm{mg} / \mathrm{kg}$ did not cause physiological abnormalities on cardiovascular system. Accordingly, these results provided the safety information of $T$. camphoratus extract for human consumption.

Keywords: Taiwanofungus camphoratus; hERG; Central Nervous System; Respiratory System; Cardiovascular System

\section{Introduction}

Taiwanofungus camphoratus (syn. Antrodia cinnamomea, Antrodia camphorata) is an edible and medicinal mushroom originating in Taiwan. Taiwan aborigines have commonly used the fruiting body of $T$. camphoratus as folk medicine for health promotion and treating liver disease, drug and food intoxication, hypertension and cancer [1,2]. Many pharmacologic studies have noted that T. camphoratus possessed a variety of biological activities including anti-oxidant, anticancer, liver protection, anti-inflammation, and immunomodulatory effects [3-11]. Additionally, many bioactive components of $T$. camphoratus have been identified, including terpenoids, polysaccharides, benzenoids, lignans, nucleic acid, benzoquinone 


\section{Advances in Clinical Toxicology}

derivatives, steroids, and maleic/succinic acid derivatives $[1,12]$. For the demand of market, it has been developed many kinds of cultivation methods to produce $T$. camphoratus including liquid fermentation, solid-state culture, cut wood culture, and dish culture. The components of T. camphoratus will depend on the culture techniques.

With the wild applications of T. camphoratus used in health food supplements in Taiwan, the safety issue of $T$. camphoratus has become increasingly important to consumers. Several toxicological studies have been done to support the safety of T. camphoratus. In 2013, Chang et al [13], reported that no abnormal findings were observed in male and female mice up to $1666.67 \mathrm{mg} / \mathrm{kg}$. Huang et al [14], demonstrated that under the dosage of $6 \mathrm{~g} / \mathrm{kg}$ of $T$. camphoratus showed no sub-chronic toxicity and teratogenicity in SD rats. Our previous studies also found the health food product "Leader Deluxe Antrodia cinnamomea" and "Leader Antrodia cinnamomea capsule" have no obvious toxic evidences in rats at dose of 2800 and $2500 \mathrm{mg} / \mathrm{kg}$, respectively $[15,16]$. The maximum tolerated dose (MTD) of the solid-state cultivated mycelial powder of Antrodia cinnamomea (LE-SC) was greater than $13.3 \mathrm{~g} / \mathrm{kg}$ bw and 90 days repeated dose oral toxicity studies also showed no significant toxicity signs in both male and female rats up to the dose of $7.6 \mathrm{~g} / \mathrm{kg} \mathrm{bw}$ [17].

T. camphoratus extract was composed of extract from cut-log cultivated fruiting body and solid-state culture of T. camphoratus. The previous studies from our laboratory revealed T. camphoratus extract showed no toxicity evidences at dose of $1700 \mathrm{mg} / \mathrm{kg}$ in 90 and 180 days repeated oral dose toxicity studies in rats (data not published). In this article, we evaluated the effects of $T$. camphoratus extract in in vitro primary cardiovascular test (hERG test) and in vivo core batteries of safety pharmacology studies on central nervous, respiratory and cardiovascular systems. All studies were designed according to the suggestions of "ICH (2001) S7A Safety Pharmacology Studies for Human Pharmaceuticals". This is the first study to explore the safety pharmacology effects of T. camphoratus and the results would provide more safety evidences for T. camphoratus.

\section{Material and Methods}

\section{Test Substance}

T. camphoratus extract, LEAC-102, was composed of extracts from cut-log cultivated fruiting body and solidstate culture of T. camphoratus that provided by Taiwan Leader Biotech Corp. (Taipei, Taiwan).

\section{Evaluation on hERG Channel Current}

Cell culture: Human ether-a-go-go-related gene transfected human embryonic kidney 293 cells (hERG transfected HEK293 Cells) were obtained from the University of Wisconsin (Madison, WI). The cells were maintained and passaged in Minimum Essential Medium (Sigma-Aldrich, St. Louis, MO) supplemented with $10 \%$ heat-inactivated fetal bovine serum, 100 units $/ \mathrm{mL}$ penicillin-streptomycin, $1 \mathrm{mmol} / \mathrm{L}$ sodium pyruvate, MEM non-essential amino acids (all supplements were from Life Technologies Corporation, Carlsbad, CA) and 400 $\mu \mathrm{g} / \mathrm{mL}$ G 418 (Geneticin; Sigma-Aldrich, St. Louis, MO). The cells were cultured in a $5 \% \mathrm{CO}_{2}$ incubator at $37^{\circ} \mathrm{C}$.

Electrophysiological experiments: The tests were conducted with the whole-cell patch-clamp method at physiological temperature $\left(37 \pm 1.0^{\circ} \mathrm{C}\right)$. T. camphoratus extract was dissolved in dimethylfloxide (DMSO) and subsequently prepared by diluting the DMSO solutions with the superfusing solution $(137 \mathrm{mmol} / \mathrm{L} \mathrm{NaCl}, 4$ $\mathrm{mmol} / \mathrm{L} \mathrm{KCl}, 1.8 \mathrm{mmol} / \mathrm{L} \mathrm{CaCl}, 1 \mathrm{mmol} / \mathrm{L} \mathrm{MgCl}_{2}, 10$ $\mathrm{mmol} / \mathrm{L}$ HEPES, and $10 \mathrm{mmol} / \mathrm{L} \mathrm{D}(+)$-glucose, $\mathrm{pH}$ adjusted to 7.4 with $\mathrm{NaOH}$ ) to obtain the test solutions of 5,10 , and $25 \mu \mathrm{g} / \mathrm{mL}$. The test solutions were filtered through a membrane filter $(0.5 \mu \mathrm{m}$; PTFE Hydrophilic membrane; Advantec Tokyo Kaisha, Ltd.) to remove fibrillike forms undissolved in the superfusing solution. The resultant solutions were used for the hERG-current measurement. The peak amplitude of the hERG tail currents was measured from 4 individual cells, which were assigned to each experimental group including test solutions at the respective concentrations $(5,10$, and 25 $\mu \mathrm{g} / \mathrm{mL}$ ), $0.5 \mathrm{vol} \%$ DMSO (vehicle control) or $0.1 \mu \mathrm{mol} / \mathrm{L} \mathrm{E}$ 4031 (positive control). The pipette solution was composed of $130 \mathrm{mmol} / \mathrm{L} \mathrm{KCl}, 1 \mathrm{mmol} / \mathrm{L} \mathrm{MgCl}_{2}, 5 \mathrm{mmol} / \mathrm{L}$ EGTA, $10 \mathrm{mmol} / \mathrm{L}$ HEPES, and $5 \mathrm{mmol} / \mathrm{L}$ MgATP (pH adjusted to 7.2 with $\mathrm{KOH}$ ).

The hERG currents passing through the cell membrane were measured under voltage clamp mode by the wholecell patch-clamp technique. A schematic diagram of the voltage protocol to elicit the hERG tail current is shown below (Figure 1); the membrane potential of the cell was held at $-80 \mathrm{mV}$, and depolarizing step pulses were given every 15 seconds to elicit the hERG tail current. The effects of the vehicle control, test substance, or positivecontrol substance on the hERG current were determined by changes in the peak amplitude of the tail current elicited by a partially repolarizing step pulse from $+20 \mathrm{mV}$ to $-50 \mathrm{mV}$ for 500 milliseconds following a depolarizing step pulse from the holding potential of $-80 \mathrm{mV}$ to +20 $\mathrm{mV}$ for 500 milliseconds. The peak value of the tail 


\section{Advances in Clinical Toxicology}

current was computed based on the holding current. After confirming a stable baseline for the peak tail currents, the test solution was applied to the cell for 11 mins at a flow rate of $5 \mathrm{~mL} / \mathrm{min}$ with a peristaltic pump (WM-120S/DV; Watson-Marlow Limited, Falmouth, UK).

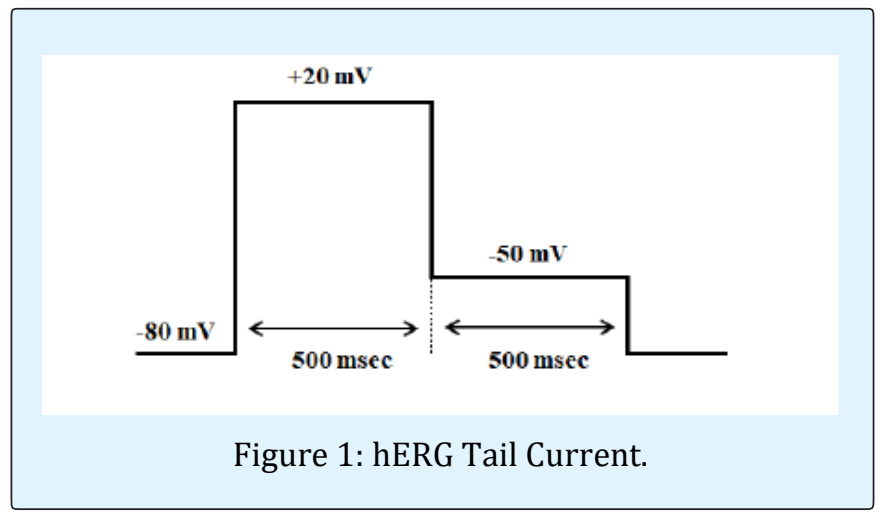

Data acquisition and analysis: The hERG currents were measured with an amplifier (Axopatch 200B; Molecular Devices, LLC., Sunnyvale, CA). Electric signals were recorded onto computer hard drive by software (pCLAMP 10; Molecular Devices, LLC., Sunnyvale, CA). The peak tail currents obtained before and 11 mins after beginning the application were compared, and the change rate (suppression rate) was calculated. The suppression rate in each cell was compensated for by the mean suppression rate in the vehicle-control group with the formula described below. Effects of the test substance and positive-control substance were evaluated with the compensated suppression rates.

- $\mathrm{X}$ : Suppression rate $(\%)$

$\mathrm{X}=[(\mathrm{A}-\mathrm{B}) / \mathrm{A}] \times 100$

A: Peak tail current in each cell immediately before application

B: Peak tail current in each cell at completion of application

-Xc: Compensated suppression rate (\%)

$\mathrm{Xc}=[(\mathrm{A}-\mathrm{B}) /(100-\mathrm{B})] \times 100$

A: Suppression rate in each cell (\%)

B: Mean suppression rate in vehicle-control group (\%)

The data are represented as mean \pm standard deviation (SD) and analyzed using one-way analysis of variance (one-way ANOVA) or student's t-test (SAS®, Ver. 9.3; SAS Institute Japan Ltd. and EXSUS, Ver. 8.0; CAC Croit Corporation). The significance levels were defined at $p<0.05$.

\section{Evaluation of Central Nervous System (CNS) in Rats}

The 8 weeks old Sprague Dawley (SD) (BioLASCO, Taiwan Co. Ltd.) rats were randomly divided into four groups with 10 males and 10 females in each group. The animals were housed in the AAALAC International accredited facility of Level Biotech. Inc. under $12 \mathrm{~h}$ light $/ 12 \mathrm{~h}$ dark cycle and the temperature of animal room was at $19.7-20.0^{\circ} \mathrm{C}$ with relative humidity $45.9-63.0 \%$. The test article, $T$. camphoratus extract, was suspended in water for injection (WFI) to obtain dosing solutions. The dosing solutions were orally administered to animals at dose of $0,170,1700$ and $3400 \mathrm{mg} / \mathrm{kg}$ for male rats and 0 , 170,850 and $1700 \mathrm{mg} / \mathrm{kg}$ for female rats. The control group was administrated with WFI only. The motor activity was conducted on all animals at pre-dose, $30 \pm 5$ mins post-dose and $24 \pm 2 \mathrm{~h}$ post-dose and the functional observation battery (FOB) was performed at pre-dose, 1.5 $\pm 0.5 \mathrm{~h}$ post-dose and $24 \pm 2 \mathrm{~h}$ post-dose. The FOB was performed of all animals including cage-side observation and handling observation (the testing parameters were including lethality, convulsion, tremor, straub tail, sedation, excitation, abnormal gait, jumps, loss of balance, motor incoordination, fore-paw treading, abnormal writhes, piloerection, stereotypies, head twitches, scratching, respiration, fear, touch response, sedation intensity, excitation intensity, aggressiveness, right reflex, ptosis, exophthalmia, grip strength, akinesia, catalepsy, corneal reflex, analgesia, defecation, salivation, lacrimation, pupillary light reflex and body temperature). All data were calculated and expressed as mean \pm SD or percentage. Comparisons of parametric data collected from treated and control groups were performed by oneway ANOVA, followed by Dunnett's method (SPSS, Ver. 12.0). Non-parametric data was analyzed by KruskalWallis nonparametric ANOVA method. The significance level was defined at $p<0.05$. The study was approved by the Institutional Animal Care and Use Committee (IACUC number: 170206-02).

\section{Evaluation of Respiratory System in Rats}

The 8-9 weeks old SD (BioLASCO, Taiwan Co. Ltd.) rats were randomly divided into four groups with 10 males and 10 females in each group. The animals were also housed in Level Biotech. Inc. under $12 \mathrm{~h}$ light/12h dark cycle and the temperature of animal room was at 19.7 $20.0^{\circ} \mathrm{C}$ with relative humidity $45.7-61.9 \%$. The test article, T. camphoratus extract, was suspended in WFI to obtain dosing solutions. The dosing solutions were orally administered to animals at dose of $0,170,1700$ and 3400 $\mathrm{mg} / \mathrm{kg}$ for male rats and $0,170,850$ and $1700 \mathrm{mg} / \mathrm{kg}$ for 
female rats. The control group was administrated with WFI only. The respiratory parameters were detected on all animals before study for $1 \mathrm{~h}$ recording as baseline, $4 \mathrm{~h}$ recording continuously after dosing and the 24 to $25 \mathrm{~h}$ recording after dosing. The whole-body plethysmography (emka TECHNOLOGIES, Paris, France) was used for detecting the respiratory parameters in freely moving animals. The parameters included inspiratory time, expiration time, peak inspiratory flow, peak expiratory flow, tidal volume, expired volume, relaxation time, minute volume, frequency of breathing, end-inspiratory pause, end-expiratory pause, enhanced pause and midexpiratory flow. All study data acquisition and analysis were operated under iox software system (emka TECHNOLOGIES, Paris, France) and an average value of each selected parameter was calculated from detectable peaks at $1 \mathrm{~min}$ interval. The data for each respiratory parameter was calculated and presented as baseline value (one hour recording data prior to dosing), four hours continuous recording data at one hour interval after dosing and 24 to $25 \mathrm{~h}$ recording after dosing (denoted as the $24^{\text {th }} \mathrm{h}$ time point). All data were expressed as mean \pm SD and analyzed by one-way ANOVA, followed by Dunnett's method (SPSS, Ver. 12.0). Besides, an additional paired $t$-test was used if the ANOVA results were statistically significant. The significance level was defined at $p<0.05$. The study was approved by the Institutional Animal Care and Use Committee (IACUC number: 180101).

\section{Evaluation of Cardiovascular System in Beagle dogs}

The 6 months old beagle dogs (Covance Inc., Cumberland, VA) were housed in the AAALAC International accredited facility of Level Biotech. Inc. under $12 \mathrm{~h}$ light/12h dark cycle and the temperature of animal room was at $19.2-22.1^{\circ} \mathrm{C}$ with relative humidity 43.7-67.2\%. Total six beagle dogs ( 3 male and 3 female) were used and treated with vehicle control (Empty Porcine Hard Gelatin Capsules) first and following treated with each dosage of $T$. camphoratus extract $(54,540,1000$ $\mathrm{mg} / \mathrm{kg}$ ) in gelatin capsules with at least 1-week washout period between treatments. The emkaPACK4G noninvasive telemetry system (emka TECHNOLOGIES, Paris, France) was used for detecting the cardiovascular parameters in freely moving animals. The parameters included RR interval, PR interval, $\mathrm{P}$ wave duration, QRS wave interval, QT interval, QTcB (Bazett's method), QTcF (Fridericia's method), heart rate, diastolic arterial pressure, systolic arterial pressure and mean arterial pressure. The electrocardiograms parameters were detected on all animals during pre-dose period for $1 \mathrm{~h}$ recording as baseline, and $24 \mathrm{~h}$ recording continuously after each dosage. The tail artery blood pressure was measured (ecgAUTO software NIBP) during the pre-dose period, $0-4^{\text {th }}$ and $23^{\text {th }}-24^{\text {th }} \mathrm{h}$ post-dosing periods. All study data acquisition was operated under iox software system (emka TECHNOLOGIES, Ver. 2.9.4.25). Study data analysis was operated under ecgAUTO software system (emka TECHNOLOGIES, Ver. 3.3.0.21). All ECG parameters were extracted from the lead II configuration. All data were expressed as mean \pm SD and analyzed by one-way ANOVA, followed by Dunnett's method (SPSS, Ver. 12.0). Besides, an additional paired $t$-test was used if the ANOVA results were statistically significant. The significance level was defined at $p<0.05$. The study was approved by the Institutional Animal Care and Use Committee (IACUC number: 171106).

\section{Results and Discussion}

\section{Effects of $T$. camphoratus Extract on hERG Current in HEK293 Cells}

The effects of the soluble fraction of T. camphoratus extract on hERG current were shown in Figures $2 \& 3$. The hERG-current-suppression rates, compensated for by the vehicle-control group rate $(11.9 \%)$, were $2.6 \%, 4.7 \%$, and $5.2 \%$ at dose of 5,10 , and $25 \mu \mathrm{g} / \mathrm{mL}$, respectively. There was no statistically significant difference between $T$. camphoratus extract groups and vehicle-control group. A hERG channel inhibitor, E-4031, was used at $0.1 \mu \mathrm{mol} / \mathrm{L}$ as a positive-control substance, and its hERG-currentsuppression rate, compensated for by the mean suppression rate in the vehicle-control group, was $86.3 \%$. This rate was statistically significant when compared to the vehicle-control group, thereby confirming the validity of this experimental system to evaluate the suppressive effects of the test substance on the hERG current. However, T. camphoratus extract was a kind of herb material and couldn't completely dissolve in DMSO solution. The test solutions were filtered through a $0.5 \mu \mathrm{m}$ PTFE Hydrophilic membrane to remove a few fibril-like forms for prevention of interference the experiments. Overall, T. camphoratus extract had no significant effect on the hERG current at nominal concentrations of up to $25 \mu \mathrm{g} / \mathrm{mL}$, which were based on the actual weight of the test substance in preparation of the DMSO solution, under the conditions of this study. 


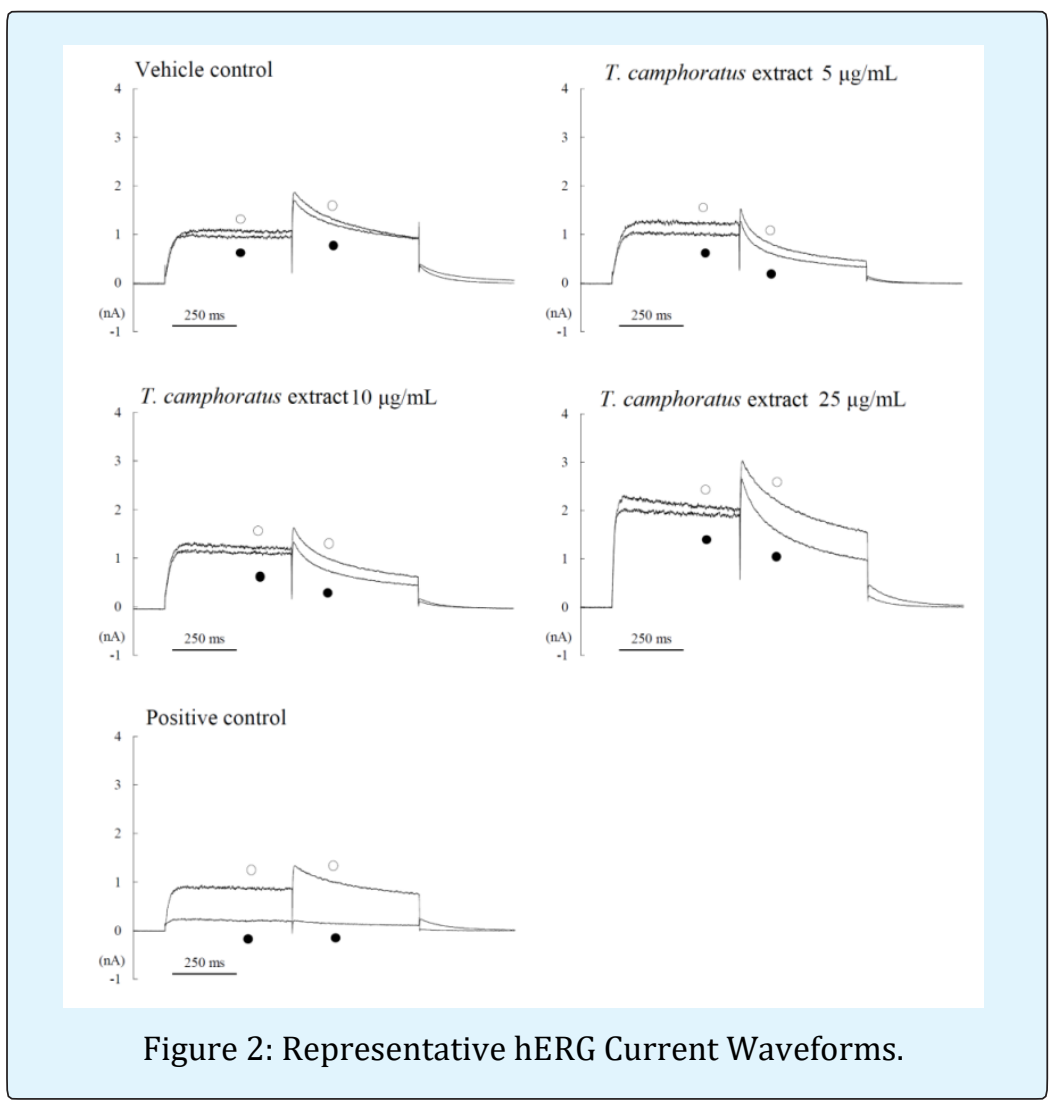

hERG-current waveforms recorded from a single HEK293 cell before (open circle) and 11 minutes after initiating the application (closed circle) are superimposed.

Vehicle control: 0.5 -vol\% DMSO; Positive control: 0.1 $\mu \mathrm{mol} / \mathrm{L} \mathrm{E}-4031$.

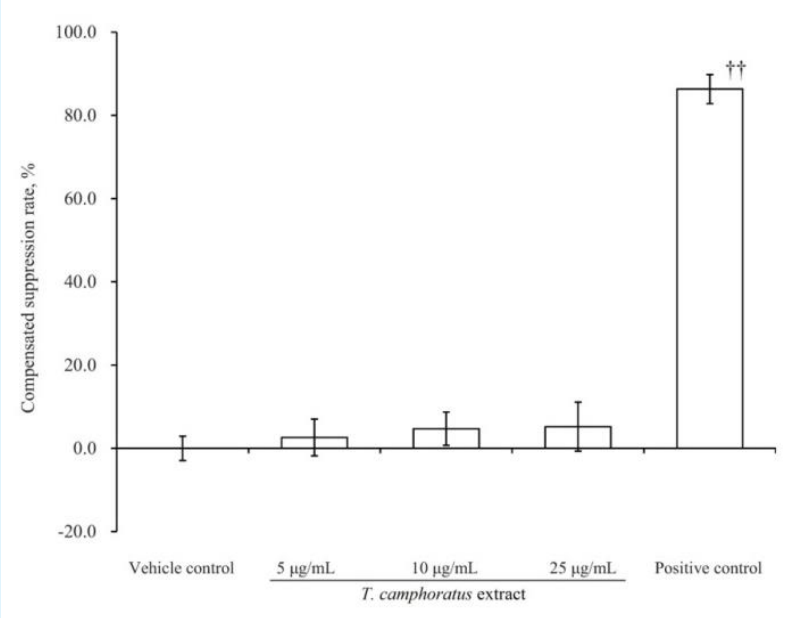

Figure 3: Effects of T. Camphoratus Extract on Tail Current from hERG Channels Expressed in HEK293 Cells. 
No significant suppressive effect of $T$. camphoratus extract on the hERG current. The mean suppression rate compensated for by $11.9 \%$, the mean suppression rate in the vehicle-control group (0.5-vol\% DMSO), 11 minutes after application was as follows: $2.6 \% \pm 4.4 \%$ at $5 \mu \mathrm{g} / \mathrm{mL}$, $4.7 \% \pm 4.0 \%$ at $10 \mu \mathrm{g} / \mathrm{mL}$, and $5.2 \% \pm 5.9 \%$ at $25 \mu \mathrm{g} / \mathrm{mL}$. The mean compensated suppression rate in the positivecontrol group $(0.1 \mu \mathrm{mol} / \mathrm{L}$ E-4031) was $86.3 \% \pm 3.5 \%$. Each column represents the mean \pm SD $(n=4)$. No statistically significant difference was noted among the test substance and vehicle-control groups, one-way ANOVA, ${ }^{+\dagger} p<0.01$, Student's $t$-test.

\section{Effects of T. camphoratus Extract on Central Nervous System (CNS) in Rats}

\begin{tabular}{|c|c|c|c|c|c|}
\hline Parameters & Gender & Dose (mg/kg) & Pre-dose & $30 \mathrm{~min}$ & $24 \mathrm{~h}$ \\
\hline \multicolumn{6}{|c|}{ Average speed (cm/sec.) } \\
\hline & \begin{tabular}{|l|} 
Male \\
\end{tabular} & 0 & $5.424 \pm 0.966$ & $4.183 \pm 1.034$ & $4.203 \pm 1.355$ \\
\hline & & 170 & $5.315 \pm 0.597$ & $4.271 \pm 0.816$ & $4.099 \pm 1.161$ \\
\hline & & 1700 & $5.502 \pm 0.898$ & $4.305 \pm 0.644$ & $4.797 \pm 0.741$ \\
\hline & & 3400 & $5.338 \pm 1.012$ & $4.973 \pm 0.682$ & $4.230 \pm 0.772$ \\
\hline & Female & 0 & $5.430 \pm 0.624$ & $4.113 \pm 0.952$ & $4.504 \pm 0.895$ \\
\hline & & 170 & $5.382 \pm 1.720$ & $4.056 \pm 1.256$ & $4.824 \pm 1.265$ \\
\hline & & 850 & $5.478 \pm 1.328$ & $4.231 \pm 1.104$ & $4.615 \pm 1.346$ \\
\hline & & 1700 & $5.288 \pm 1.641$ & $4.198 \pm 0.850$ & $5.256 \pm 1.402$ \\
\hline \multicolumn{6}{|c|}{ Max speed (cm/sec.) } \\
\hline & Male & 0 & $41.996 \pm 10.904$ & $34.101 \pm 11.971$ & $27.244 \pm 12.993$ \\
\hline & & 170 & $40.515 \pm 7.521$ & $31.513 \pm 5.760$ & $28.439 \pm 12.583$ \\
\hline & & 1700 & $42.572 \pm 15.210$ & $33.105 \pm 7.395$ & $37.382 \pm 8.473$ \\
\hline & & 3400 & $46.482 \pm 8.687$ & $41.703 \pm 10.611$ & $38.373 \pm 11.421$ \\
\hline & Female & 0 & $51.748 \pm 8.954$ & $40.166 \pm 17.737$ & $43.893 \pm 18.941$ \\
\hline & & 170 & $45.023 \pm 16.185$ & $35.371 \pm 22.634$ & $40.370 \pm 17.980$ \\
\hline & & 850 & $46.491 \pm 8.777$ & $37.963 \pm 15.302$ & $45.788 \pm 15.866$ \\
\hline & & 1700 & $59.697 \pm 34.940$ & $48.554 \pm 19.337$ & $55.333 \pm 8.855$ \\
\hline \multicolumn{6}{|c|}{ Total distance $(\mathrm{cm})$} \\
\hline & Male & 0 & $3270.646 \pm 1149.900$ & $2458.525 \pm 1023.302$ & $2642.129 \pm 1344.129$ \\
\hline & & 170 & $3346.333 \pm 722.597$ & $2657.786 \pm 712.840$ & $2840.399 \pm 1169.978$ \\
\hline & & 1700 & $3967.793 \pm 1340.863$ & $2854.312 \pm 1062.888$ & $3574.599 \pm 877.609$ \\
\hline & & 3400 & $3862.154 \pm 1466.586$ & $2974.510 \pm 1011.123$ & $2900.317 \pm 1037.412$ \\
\hline & Female & 0 & $2994.735 \pm 712.093$ & $2152.536 \pm 658.927$ & $2767.476 \pm 1208.073$ \\
\hline & & 170 & $3689.441 \pm 1805.136$ & $2503.654 \pm 740.367$ & $3344.445 \pm 1093.070$ \\
\hline & & 850 & $3734.458 \pm 2002.942$ & $2376.107 \pm 1022.169$ & $3343.206 \pm 1081.230$ \\
\hline & & 1700 & $3521.432 \pm 1479.436$ & $2502.781 \pm 645.590$ & $3649.590 \pm 1210.729$ \\
\hline
\end{tabular}

Table 1: Effect of T. Camphoratus Extract on Motor Activity in Rats.

All data presented as mean \pm SD.

Lin JY and Chiu E. Preclinical Safety Pharmacology Studies of Taiwanofungus camphoratus Camphoratus Extract. Adv Clin Toxicol 2019, 4(1): 000146.
The male and female rats received T. camphoratus extract at doses up to $3400 \mathrm{mg} / \mathrm{kg}$ and $1700 \mathrm{mg} / \mathrm{kg}$, respectively, showed no significant effects on CNS. Results of motor activity and functional observational battery (FOB) responses were shown in Tables $1 \& 2$. No treatment related changes were observed in motor activity at pre-dose period, 30 mins and $24 \mathrm{~h}$ post-dosing periods. In FOB measurements, the value of grip strength in high-dose $(3400 \mathrm{mg} / \mathrm{kg})$ males was statistically significantly higher than vehicle control group at $24 \mathrm{~h}$ post-dosing period. However, this change was considered was not correlated with other parameters of motor activity and FOB. Based on the results, T. camphoratus extract showed no adverse effects on CNS in rats that could provide safety information for human exposure. incidental and unrelated to treatment because the change 
Advances in Clinical Toxicology

\begin{tabular}{|c|c|c|c|c|c|}
\hline Parameters & Gender & Dose (mg/kg) & Pre-dose & $1.5 \mathrm{~h}$ & $24 \mathrm{~h}$ \\
\hline \multicolumn{6}{|c|}{ Respiration (breath per min.) } \\
\hline & Male & 0 & $126.6 \pm 14.0$ & $106.8 \pm 7.4$ & $111.6 \pm 9.9$ \\
\hline & & 170 & $125.4 \pm 10.8$ & $115.8 \pm 8.0$ & $117.6 \pm 9.5$ \\
\hline & & 1700 & $120.6 \pm 7.7$ & $110.4 \pm 11.7$ & $117.0 \pm 12.1$ \\
\hline & & 3400 & $122.4 \pm 13.6$ & $112.8 \pm 6.8$ & $112.2 \pm 13.6$ \\
\hline & Female & 0 & $114.6 \pm 11.1$ & $96.0 \pm 16.2$ & $94.2 \pm 9.8$ \\
\hline & & 170 & $119.4 \pm 16.1$ & $96.6 \pm 10.0$ & $88.2 \pm 13.9$ \\
\hline & & 850 & $105.6 \pm 12.1$ & $99.6 \pm 13.0$ & $93.0 \pm 11.7$ \\
\hline & & 1700 & $108.0 \pm 12.0$ & $97.8 \pm 12.7$ & $92.4 \pm 11.7$ \\
\hline \multicolumn{6}{|c|}{ Grip strength (kg) } \\
\hline & Male & 0 & $0.63 \pm 0.07$ & $0.69 \pm 0.06$ & $0.66 \pm 0.07$ \\
\hline & & 170 & $0.69 \pm 0.07$ & $0.66 \pm 0.11$ & $0.68 \pm 0.04$ \\
\hline & & 1700 & $0.63 \pm 0.08$ & $0.69 \pm 0.06$ & $0.69 \pm 0.06$ \\
\hline & & 3400 & $0.66 \pm 0.07$ & $0.68 \pm 0.06$ & $0.74 \pm 0.05^{*}$ \\
\hline & Female & 0 & $0.60 \pm 0.07$ & $0.65 \pm 0.08$ & $0.65 \pm 0.07$ \\
\hline & & 170 & $0.59 \pm 0.06$ & $0.62 \pm 0.06$ & $0.69 \pm 0.06$ \\
\hline & & 850 & $0.62 \pm 0.06$ & $0.66 \pm 0.05$ & $0.68 \pm 0.04$ \\
\hline & & 1700 & $0.58 \pm 0.06$ & $0.64 \pm 0.08$ & $0.68 \pm 0.08$ \\
\hline \multicolumn{6}{|l|}{ Defecation } \\
\hline & Male & 0 & $0.5 \pm 0.5$ & $1.0 \pm 0.0$ & $0.7 \pm 0.5$ \\
\hline & & 170 & $0.8 \pm 0.4$ & $0.7 \pm 0.5$ & $0.4 \pm 0.5$ \\
\hline & & 1700 & $0.5 \pm 0.5$ & $1.0 \pm 0.0$ & $0.4 \pm 0.5$ \\
\hline & & 3400 & $0.5 \pm 0.5$ & $0.6 \pm 0.5$ & $0.5 \pm 0.5$ \\
\hline & Female & 0 & $0.7 \pm 0.5$ & $0.8 \pm 0.4$ & $1.0 \pm 0.0$ \\
\hline & & 170 & $0.9 \pm 0.3$ & $0.9 \pm 0.3$ & $0.8 \pm 0.4$ \\
\hline & & 850 & $0.9 \pm 0.3$ & $0.7 \pm 0.5$ & $1.0 \pm 0.0$ \\
\hline & & 1700 & $0.9 \pm 0.3$ & $0.9 \pm 0.3$ & $0.9 \pm 0.3$ \\
\hline \multicolumn{6}{|c|}{ Body temperature Scores } \\
\hline & Male & 0 & $-0.1 \pm 0.3$ & $0.0 \pm 0.0$ & $0.0 \pm 0.0$ \\
\hline & & 170 & $0.0 \pm 0.0$ & $0.0 \pm 0.0$ & $0.0 \pm 0.0$ \\
\hline & & 1700 & $0.0 \pm 0.0$ & $0.0 \pm 0.5$ & $0.0 \pm 0.0$ \\
\hline & & 3400 & $-0.1 \pm 0.3$ & $0.0 \pm 0.0$ & $0.0 \pm 0.0$ \\
\hline & Female & 0 & $0.0 \pm 0.0$ & $0.0 \pm 0.0$ & $-0.2 \pm 0.4$ \\
\hline & & 170 & $0.0 \pm 0.0$ & $0.0 \pm 0.0$ & $-0.2 \pm 0.4$ \\
\hline & & 850 & $0.0 \pm 0.0$ & $0.0 \pm 0.0$ & $0.0 \pm 0.0$ \\
\hline & & 1700 & $0.0 \pm 0.0$ & $0.0 \pm 0.0$ & $-0.1 \pm 0.3$ \\
\hline
\end{tabular}

Table 2: Effect of T. Camphoratus Extract on Functional Observation Battery in Rats.

All data presented as mean \pm SD.

${ }^{*} p<0.05$ compared to vehicle control group.

\section{Effects of T. camphoratus Extract on Respiratory System in Rats}

The male and female rats received T. camphoratus extract at doses up to $3400 \mathrm{mg} / \mathrm{kg}$ and $1700 \mathrm{mg} / \mathrm{kg}$, respectively, showed no significant effects on respiratory system. There was no statistical difference noted in all respiratory parameters among all treated groups during each time period (Table 3). Based on the test results, $T$. camphoratus extract administered to rats via oral gavage up to the dosage of $3400 \mathrm{mg} / \mathrm{kg}$ for males and 1700 $\mathrm{mg} / \mathrm{kg}$ for females did not cause significant abnormal respiratory effects in this study. All results generated from this study will provide safety information for human exposure. 


\section{Advances in Clinical Toxicology}

\begin{tabular}{|c|c|c|c|c|c|c|c|}
\hline \multicolumn{2}{|c|}{ Parameters Gender|Dose (mg/kg) } & Pre-dose & $1 \mathrm{~h}$ & $2 \mathrm{~h}$ & $3 \mathrm{~h}$ & $4 \mathrm{~h}$ & $24 \mathrm{~h}$ \\
\hline \\
\hline \begin{tabular}{|l|l|} 
& Male \\
\end{tabular} & \begin{tabular}{l|l}
0 \\
0
\end{tabular} & $200.34 \pm 25.95$ & $169.74 \pm 27.42$ & $218.75 \pm 24.24$ & $216.94 \pm 14.52$ & $219.42 \pm 22.57$ & $201.12 \pm 26.08$ \\
\hline & 170 & $216.34 \pm 22.56$ & $169.43 \pm 40.69$ & $209.00 \pm 33.81$ & $214.32 \pm 20.86$ & $211.94 \pm 36.31$ & $217.51 \pm 19.92$ \\
\hline & 1700 & $206.80 \pm 17.05$ & $179.65 \pm 27.40$ & $209.31 \pm 27.36$ & $212.86 \pm 27.88$ & $210.93 \pm 34.55$ & $206.79 \pm 18.14$ \\
\hline & 3400 & $209.92 \pm 26.24$ & $161.14 \pm 28.06$ & $210.60 \pm 24.84$ & $225.75 \pm 20.21$ & $220.75 \pm 26.18$ & $215.81 \pm 27.27$ \\
\hline Female & 0 & $187.38 \pm 58.44$ & $178.32 \pm 27.67$ & $260.15 \pm 28.83$ & $248.82 \pm 22.91$ & $254.34 \pm 36.85$ & $240.97 \pm 36.48$ \\
\hline & 170 & $161.36 \pm 44.39$ & $165.94 \pm 36.96$ & $232.59 \pm 32.92$ & $259.87 \pm 37.36$ & $262.10 \pm 24.54$ & $230.52 \pm 51.11$ \\
\hline & 850 & $188.80 \pm 40.22$ & $175.45 \pm 34.88$ & $241.26 \pm 45.74$ & $250.23 \pm 30.97$ & $255.69 \pm 22.14$ & $238.89 \pm 23.05$ \\
\hline & 1700 & $189.44 \pm 42.26$ & $207.44 \pm 40.58$ & $248.24 \pm 25.60$ & $235.01 \pm 59.60$ & $244.48 \pm 47.57$ & $249.50 \pm 29.30$ \\
\hline \multicolumn{8}{|c|}{ Expiration Time (msec) } \\
\hline \begin{tabular}{l|l|} 
& Male \\
\end{tabular} & 0 & $284.01 \pm 37.39$ & $264.67 \pm 36.04$ & $313.36 \pm 37.68$ & $314.75 \pm 33.81$ & $324.63 \pm 42.57$ & $295.30 \pm 34.41$ \\
\hline & 170 & $290.18 \pm 31.09$ & $248.84 \pm 46.29$ & $298.65 \pm 33.30$ & $305.01 \pm 36.65$ & $303.86 \pm 50.67$ & $305.75 \pm 29.16$ \\
\hline & 1700 & $274.96 \pm 25.10$ & $262.90 \pm 40.88$ & $294.78 \pm 33.96$ & $295.36 \pm 35.08$ & $299.09 \pm 33.90$ & $289.19 \pm 26.32$ \\
\hline & 3400 & $286.84 \pm 54.55$ & $252.51 \pm 46.63$ & $294.48 \pm 43.32$ & $306.74 \pm 46.06$ & $306.30 \pm 45.20$ & $299.14 \pm 50.31$ \\
\hline Female & 0 & $329.39 \pm 97.33$ & $323.53 \pm 68.06$ & $456.26 \pm 78.57$ & $421.07 \pm 44.61$ & $436.07 \pm 81.17$ & $413.01 \pm 73.20$ \\
\hline & 170 & $274.69 \pm 78.53$ & $291.07 \pm 51.51$ & $410.96 \pm 80.30$ & $437.24 \pm 72.00$ & $449.93 \pm 77.51$ & $386.97 \pm 93.80$ \\
\hline & 850 & $296.45 \pm 61.10$ & $300.88 \pm 52.91$ & $394.30 \pm 52.12$ & $397.16 \pm 44.19$ & $407.98 \pm 44.11$ & $392.59 \pm 31.93$ \\
\hline & 1700 & $301.30 \pm 65.78$ & $383.85 \pm 134.95$ & $431.83 \pm 123.98$ & $379.18 \pm 86.56$ & $386.96 \pm 68.95$ & $398.67 \pm 46.46$ \\
\hline \multicolumn{8}{|c|}{ Peak Inspiratory Flow (mL/s) } \\
\hline \begin{tabular}{|l|l|} 
& Male \\
\end{tabular} & 0 & $10.93 \pm 2.90$ & $13.53 \pm 2.36$ & $8.22 \pm 1.98$ & $8.66 \pm 1.41$ & $8.51 \pm 1.63$ & $11.07 \pm 3.01$ \\
\hline & 170 & $8.93 \pm 2.29$ & $13.86 \pm 4.77$ & $9.35 \pm 3.57$ & $8.42 \pm 2.28$ & $9.56 \pm 4.31$ & $9.35 \pm 2.61$ \\
\hline & 1700 & $9.68 \pm 1.41$ & $12.67 \pm 1.88$ & $9.83 \pm 2.18$ & $9.28 \pm 2.89$ & $9.55 \pm 2.36$ & $9.93 \pm 1.01$ \\
\hline & 3400 & $9.26 \pm 1.79$ & $13.99 \pm 2.21$ & $9.04 \pm 2.05$ & $7.66 \pm 1.39$ & $7.79 \pm 1.63$ & $10.11 \pm 4.83$ \\
\hline Female & 0 & $13.21 \pm 3.92$ & $13.17 \pm 2.24$ & $7.07 \pm 1.25$ & $7.84 \pm 1.52$ & $7.37 \pm 2.02$ & $8.00 \pm 2.44$ \\
\hline & 170 & $15.38 \pm 6.75$ & $14.03 \pm 6.09$ & $9.34 \pm 2.72$ & $7.24 \pm 2.01$ & $7.74 \pm 4.71$ & $9.03 \pm 3.54$ \\
\hline & 850 & $13.13 \pm 3.56$ & $12.74 \pm 3.34$ & $8.35 \pm 3.51$ & $7.87 \pm 2.34$ & $7.29 \pm 1.58$ & $8.19 \pm 1.50$ \\
\hline & 1700 & $12.73 \pm 3.39$ & $10.71 \pm 3.23$ & $7.36 \pm 2.02$ & $9.30 \pm 3.64$ & $8.28 \pm 2.66$ & $7.04 \pm 1.65$ \\
\hline \multicolumn{8}{|c|}{ Peak Expiratory Flow (mL/s) } \\
\hline \begin{tabular}{|l|l|} 
& Male \\
\end{tabular} & 0 & $11.59 \pm 2.12$ & $12.39 \pm 1.00$ & $9.61 \pm 1.66$ & $9.83 \pm 1.59$ & $9.97 \pm 1.49$ & $11.93 \pm 2.78$ \\
\hline & 170 & $10.42 \pm 2.20$ & $12.77 \pm 3.37$ & $10.39 \pm 2.39$ & $9.73 \pm 2.10$ & $10.68 \pm 2.87$ & $11.07 \pm 3.05$ \\
\hline & 1700 & $10.70 \pm 1.43$ & $11.64 \pm 1.63$ & $10.45 \pm 1.60$ & $10.18 \pm 1.72$ & $10.31 \pm 1.35$ & $10.99 \pm 1.51$ \\
\hline & 3400 & $10.56 \pm 1.33$ & $12.35 \pm 1.52$ & $9.83 \pm 1.14$ & $9.22 \pm 0.89$ & $9.35 \pm 1.08$ & $11.41 \pm 3.89$ \\
\hline Female & 0 & $11.81 \pm 2.60$ & $11.28 \pm 1.86$ & $7.77 \pm 0.64$ & $8.12 \pm 1.22$ & $7.83 \pm 1.47$ & $8.61 \pm 1.33$ \\
\hline & 170 & $14.30 \pm 6.11$ & $12.50 \pm 5.96$ & $9.69 \pm 2.74$ & $8.03 \pm 2.19$ & $8.32 \pm 4.01$ & $9.26 \pm 2.03$ \\
\hline & 850 & $12.81 \pm 3.14$ & $11.05 \pm 2.28$ & $8.77 \pm 1.81$ & $8.35 \pm 1.77$ & $8.10 \pm 1.31$ & $8.85 \pm 1.09$ \\
\hline & 1700 & $12.31 \pm 2.81$ & $9.81 \pm 1.89$ & $7.84 \pm 1.92$ & $9.28 \pm 2.46$ & $8.40 \pm 1.33$ & $7.81 \pm 1.40$ \\
\hline \multicolumn{8}{|l|}{ Tidal Volume (mL) } \\
\hline \begin{tabular}{l|l} 
& Male \\
\end{tabular} & 0 & $1.12 \pm 0.20$ & $1.17 \pm 0.18$ & $1.02 \pm 0.17$ & $1.04 \pm 0.14$ & $1.03 \pm 0.13$ & $1.20 \pm 0.23$ \\
\hline & 170 & $1.03 \pm 0.23$ & $1.14 \pm 0.21$ & $1.05 \pm 0.18$ & $1.01 \pm 0.21$ & $1.04 \pm 0.21$ & $1.12 \pm 0.22$ \\
\hline & 1700 & $1.11 \pm 0.09$ & $1.22 \pm 0.15$ & $1.08 \pm 0.14$ & $1.05 \pm 0.11$ & $1.09 \pm 0.12$ & $1.16 \pm 0.18$ \\
\hline & 3400 & $1.10 \pm 0.08$ & $1.16 \pm 0.12$ & $1.01 \pm 0.11$ & $1.00 \pm 0.09$ & $1.00 \pm 0.09$ & $1.17 \pm 0.28$ \\
\hline Female & 0 & $1.00 \pm 0.05$ & $0.99 \pm 0.06$ & $0.87 \pm 0.07$ & $0.89 \pm 0.10$ & $0.87 \pm 0.07$ & $0.92 \pm 0.10$ \\
\hline & 170 & $1.02 \pm 0.31$ & $0.98 \pm 0.30$ & $0.98 \pm 0.29$ & $0.88 \pm 0.24$ & $0.90 \pm 0.28$ & $0.92 \pm 0.11$ \\
\hline & 850 & $1.04 \pm 0.10$ & $1.05 \pm 0.12$ & $0.91 \pm 0.12$ & $0.92 \pm 0.09$ & $0.91 \pm 0.07$ & $0.93 \pm 0.08$ \\
\hline & 1700 & $0.98 \pm 0.12$ & $0.95 \pm 0.14$ & $0.87 \pm 0.16$ & $0.93 \pm 0.12$ & $0.91 \pm 0.10$ & $0.87 \pm 0.11$ \\
\hline \multicolumn{8}{|c|}{ Expired Volume (mL) } \\
\hline \begin{tabular}{l|l|} 
& Male \\
\end{tabular} & 0 & $1.12 \pm 0.20$ & $1.16 \pm 0.17$ & $1.02 \pm 0.17$ & $1.05 \pm 0.14$ & $1.02 \pm 0.14$ & $1.19 \pm 0.23$ \\
\hline & 170 & $1.04 \pm 0.25$ & $1.14 \pm 0.21$ & $1.05 \pm 0.18$ & $1.02 \pm 0.21$ & $1.03 \pm 0.20$ & $1.14 \pm 0.24$ \\
\hline & 1700 & $1.13 \pm 0.09$ & $1.22 \pm 0.12$ & $1.10 \pm 0.12$ & $1.05 \pm 0.11$ & $1.09 \pm 0.13$ & $1.17 \pm 0.21$ \\
\hline
\end{tabular}




\begin{tabular}{|c|c|c|c|c|c|c|c|c|}
\hline & & 3400 & $1.10 \pm 0.08$ & $1.18 \pm 0.09$ & $1.01 \pm 0.11$ & $1.01 \pm 0.09$ & $0.99 \pm 0.09$ & $1.17 \pm 0.28$ \\
\hline & Female & 0 & $1.00 \pm 0.05$ & $1.00 \pm 0.07$ & $0.87 \pm 0.07$ & $0.88 \pm 0.11$ & $0.89 \pm 0.06$ & $0.91 \pm 0.11$ \\
\hline & & 170 & $1.03 \pm 0.31$ & $0.98 \pm 0.30$ & $0.96 \pm 0.27$ & $0.89 \pm 0.25$ & $0.91 \pm 0.28$ & $0.92 \pm 0.11$ \\
\hline & & 850 & $1.05 \pm 0.08$ & $1.05 \pm 0.12$ & $0.90 \pm 0.12$ & $0.92 \pm 0.09$ & $0.92 \pm 0.06$ & $0.93 \pm 0.08$ \\
\hline & & 1700 & $0.99 \pm 0.13$ & $0.96 \pm 0.13$ & $0.86 \pm 0.18$ & $0.96 \pm 0.10$ & $0.95 \pm 0.10$ & $0.88 \pm 0.11$ \\
\hline \multicolumn{9}{|c|}{ Relaxation Time (msec) } \\
\hline & Male & 0 & $122.26 \pm 11.22$ & $119.61 \pm 10.02$ & $129.47 \pm 13.59$ & $131.90 \pm 14.28$ & $132.31 \pm 14.27$ & $127.24 \pm 12.34$ \\
\hline & & 170 & $123.81 \pm 11.61$ & $116.87 \pm 16.00$ & $127.44 \pm 11.17$ & $128.19 \pm 10.80$ & $125.30 \pm 15.99$ & $128.15 \pm 11.72$ \\
\hline & & 1700 & $123.53 \pm 7.60$ & $124.82 \pm 18.40$ & $130.09 \pm 11.75$ & $129.98 \pm 13.69$ & $131.30 \pm 13.03$ & $126.67 \pm 8.35$ \\
\hline & & 3400 & $123.74 \pm 11.39$ & $118.98 \pm 12.42$ & $126.23 \pm 9.39$ & $131.61 \pm 9.46$ & $130.01 \pm 11.80$ & $126.89 \pm 12.30$ \\
\hline & Female & 0 & $132.33 \pm 27.18$ & $138.18 \pm 28.97$ & $177.75 \pm 40.53$ & $170.53 \pm 31.60$ & $173.17 \pm 35.63$ & $155.82 \pm 23.36$ \\
\hline & & 170 & $113.63 \pm 25.11$ & $127.74 \pm 27.73$ & $165.54 \pm 55.50$ & $179.34 \pm 62.47$ & $184.04 \pm 60.26$ & $142.38 \pm 18.61$ \\
\hline & & 850 & $123.56 \pm 19.09$ & $133.79 \pm 17.38$ & $152.94 \pm 18.85$ & $158.39 \pm 17.18$ & $160.81 \pm 15.11$ & $148.80 \pm 8.23$ \\
\hline & & 1700 & $122.02 \pm 18.37$ & $156.85 \pm 52.22$ & $189.01 \pm 90.36$ & $167.60 \pm 56.79$ & $161.13 \pm 26.39$ & $152.71 \pm 16.32$ \\
\hline \multicolumn{9}{|c|}{ Minute Volume (mL) } \\
\hline & Male & 0 & $168.58 \pm 44.00$ & $205.95 \pm 31.33$ & $125.63 \pm 29.63$ & $129.28 \pm 19.95$ & $128.45 \pm 27.36$ & $168.64 \pm 44.76$ \\
\hline & & 170 & $139.58 \pm 36.75$ & $220.04 \pm 81.29$ & $143.37 \pm 50.06$ & $131.99 \pm 35.91$ & $145.40 \pm 62.42$ & $144.06 \pm 40.28$ \\
\hline & & 1700 & $152.41 \pm 20.74$ & $196.85 \pm 30.44$ & $147.68 \pm 32.41$ & $142.00 \pm 43.18$ & $144.42 \pm 33.26$ & $154.43 \pm 16.99$ \\
\hline & & 3400 & $149.82 \pm 31.87$ & $219.80 \pm 36.34$ & $139.27 \pm 31.80$ & $120.96 \pm 21.60$ & $122.39 \pm 23.45$ & $157.52 \pm 67.16$ \\
\hline & Female & 0 & $199.42 \pm 70.03$ & $196.41 \pm 42.45$ & $92.52 \pm 22.78$ & $108.16 \pm 22.97$ & $100.79 \pm 33.96$ & $111.87 \pm 37.45$ \\
\hline & & 170 & $251.26 \pm 119.10$ & $220.78 \pm 110.35$ & $133.84 \pm 46.14$ & $99.42 \pm 29.84$ & $104.54 \pm 65.93$ & $126.00 \pm 56.07$ \\
\hline & & 850 & $207.71 \pm 65.02$ & $192.09 \pm 51.63$ & $114.62 \pm 49.39$ & $111.03 \pm 34.81$ & $101.54 \pm 24.52$ & $113.47 \pm 20.54$ \\
\hline & & 1700 & $200.40 \pm 66.01$ & $154.98 \pm 55.67$ & $100.97 \pm 34.90$ & $134.63 \pm 61.49$ & $118.07 \pm 40.20$ & $100.22 \pm 25.15$ \\
\hline \multicolumn{9}{|c|}{ Frequency of breathing (bpm) } \\
\hline & \begin{tabular}{|l|} 
Male \\
\end{tabular} & 0 & $151.26 \pm 28.28$ & $181.64 \pm 39.36$ & $124.50 \pm 21.65$ & $126.81 \pm 13.06$ & $125.47 \pm 25.24$ & $140.68 \pm 27.35$ \\
\hline & & 170 & $132.55 \pm 16.62$ & $191.63 \pm 52.49$ & $136.67 \pm 32.41$ & $131.53 \pm 25.98$ & $139.22 \pm 37.54$ & $127.31 \pm 16.17$ \\
\hline & & 1700 & $136.98 \pm 14.91$ & $170.14 \pm 30.94$ & $134.91 \pm 20.48$ & $134.08 \pm 32.16$ & $132.20 \pm 27.17$ & $133.55 \pm 16.26$ \\
\hline & & 3400 & $137.29 \pm 26.62$ & $197.79 \pm 40.82$ & $138.86 \pm 26.02$ & $121.07 \pm 17.21$ & $123.97 \pm 21.86$ & $133.03 \pm 27.51$ \\
\hline & Female & 0 & $201.60 \pm 71.84$ & $198.35 \pm 41.89$ & $103.29 \pm 23.84$ & $121.14 \pm 24.40$ & $114.68 \pm 35.22$ & $122.23 \pm 38.53$ \\
\hline & & 170 & $244.77 \pm 84.82$ & $221.74 \pm 50.13$ & $139.50 \pm 41.87$ & $112.48 \pm 22.49$ & $108.41 \pm 35.16$ & $138.24 \pm 63.74$ \\
\hline & & 850 & $202.11 \pm 58.91$ & $190.68 \pm 48.26$ & $125.20 \pm 48.49$ & $121.21 \pm 35.33$ & $109.03 \pm 20.78$ & $121.24 \pm 20.93$ \\
\hline & & 1700 & $202.13 \pm 61.13$ & $161.13 \pm 47.62$ & $115.01 \pm 31.25$ & $143.62 \pm 67.68$ & $130.99 \pm 47.60$ & $115.54 \pm 24.13$ \\
\hline \multicolumn{9}{|c|}{ End-Inspiratory Pause (msec) } \\
\hline & Male & 0 & $4.29 \pm 0.95$ & $3.58 \pm 0.65$ & $4.31 \pm 0.69$ & $4.18 \pm 0.40$ & $4.19 \pm 0.40$ & $3.83 \pm 0.57$ \\
\hline & & 170 & $4.33 \pm 0.74$ & $3.61 \pm 0.72$ & $3.84 \pm 0.73$ & $4.27 \pm 1.01$ & $4.34 \pm 1.15$ & $4.06 \pm 0.55$ \\
\hline & & 1700 & $4.36 \pm 0.54$ & $4.43 \pm 2.74$ & $4.93 \pm 1.99$ & $5.08 \pm 1.67$ & $4.50 \pm 1.20$ & $3.95 \pm 0.44$ \\
\hline & & 3400 & $3.98 \pm 1.13$ & $3.21 \pm 0.50$ & $4.36 \pm 1.25$ & $4.44 \pm 0.89$ & $4.29 \pm 1.14$ & $4.36 \pm 1.24$ \\
\hline & Female & 0 & $4.71 \pm 1.64$ & $5.55 \pm 3.71$ & $9.83 \pm 7.44$ & $9.21 \pm 6.66$ & $9.33 \pm 6.58$ & $6.21 \pm 1.66$ \\
\hline & & 170 & $3.76 \pm 1.21$ & $4.92 \pm 3.37$ & $6.02 \pm 1.71$ & $7.94 \pm 3.46$ & $7.62 \pm 3.24$ & $5.18 \pm 2.02$ \\
\hline & & 850 & $4.57 \pm 1.90$ & $4.56 \pm 1.85$ & $7.68 \pm 3.53$ & $8.09 \pm 3.50$ & $8.40 \pm 4.31$ & $5.28 \pm 1.05$ \\
\hline & & 1700 & $5.21 \pm 2.55$ & $7.42 \pm 6.04$ & $10.30 \pm 9.15$ & $9.80 \pm 8.62$ & $9.60 \pm 7.42$ & $6.06 \pm 1.96$ \\
\hline \multicolumn{9}{|c|}{ End-Expiratory Pause (msec) } \\
\hline & Male & 0 & $54.13 \pm 11.92$ & $45.13 \pm 10.78$ & $61.79 \pm 12.81$ & $60.39 \pm 11.77$ & $62.97 \pm 13.78$ & $55.86 \pm 11.24$ \\
\hline & & 170 & $57.44 \pm 9.55$ & $42.32 \pm 11.69$ & $57.14 \pm 14.39$ & $59.25 \pm 13.88$ & $58.90 \pm 12.71$ & $61.17 \pm 8.57$ \\
\hline & & 1700 & $52.54 \pm 8.60$ & $44.07 \pm 8.05$ & $54.77 \pm 9.97$ & $54.04 \pm 9.64$ & $55.46 \pm 9.48$ & $55.81 \pm 9.62$ \\
\hline & & 3400 & $55.48 \pm 17.28$ & $42.71 \pm 13.68$ & $56.03 \pm 11.79$ & $58.43 \pm 12.83$ & $58.23 \pm 12.87$ & $59.72 \pm 14.38$ \\
\hline & Female & 0 & $56.52 \pm 20.23$ & $51.48 \pm 11.57$ & $78.98 \pm 16.36$ & $73.63 \pm 9.27$ & $75.95 \pm 13.91$ & $78.04 \pm 16.29$ \\
\hline & & 170 & $49.55 \pm 19.30$ & $48.79 \pm 12.18$ & $77.44 \pm 28.06$ & $82.18 \pm 21.79$ & $83.97 \pm 16.90$ & $76.85 \pm 33.19$ \\
\hline & & 850 & $55.74 \pm 15.72$ & $47.54 \pm 10.46$ & $70.23 \pm 13.09$ & $70.93 \pm 10.14$ & $75.51 \pm 9.17$ & $75.08 \pm 7.90$ \\
\hline & & 1700 & $56.87 \pm 16.84$ & $58.59 \pm 15.98$ & $70.95 \pm 20.08$ & $63.04 \pm 22.65$ & $66.12 \pm 15.79$ & $76.93 \pm 12.53$ \\
\hline
\end{tabular}




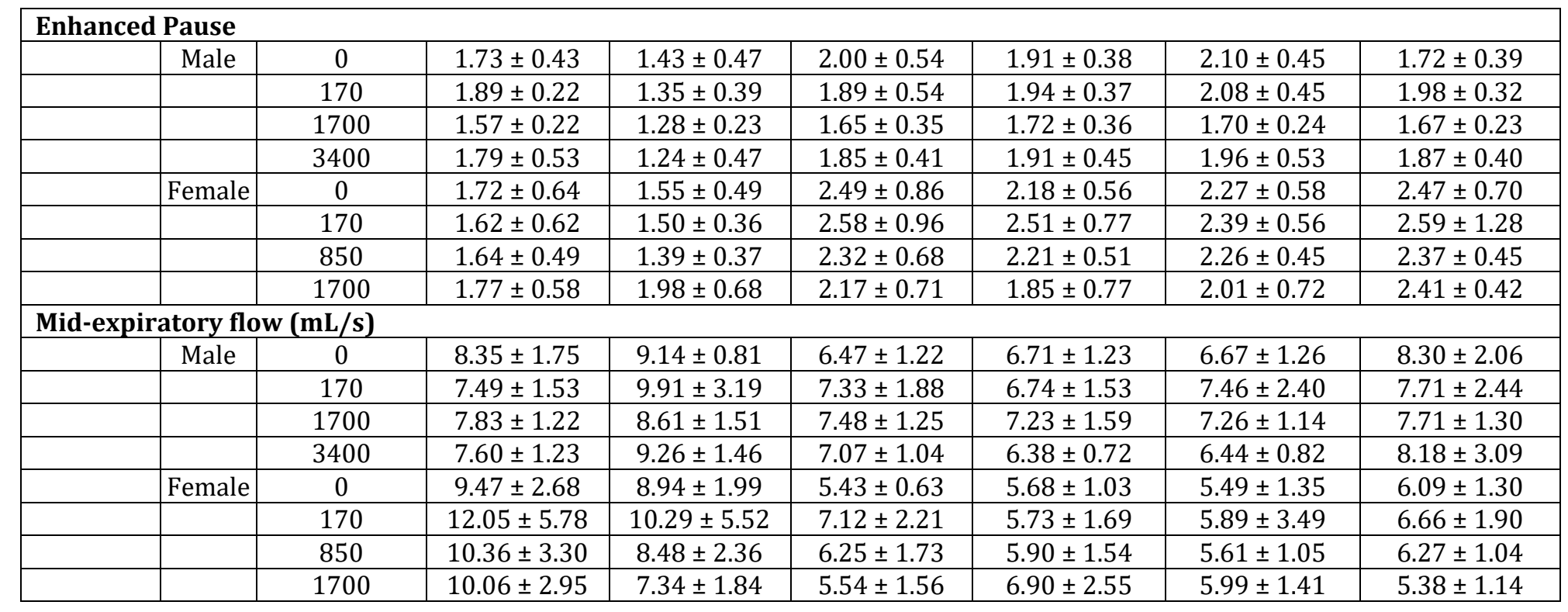

Table 3: Effect of T. camphoratus extract on respiratory parameters in rats. All data presented as mean \pm SD.

\section{Effects of T. camphoratus Extract on Cardiovascular System in Beagle Dogs}

The beagle dogs were given gelatin capsules containing T. camphoratus extract at doses of 0 (empty capsule), 54, 540, $1000 \mathrm{mg} / \mathrm{kg}$ orally and the ECG parameters and blood pressures were measured at indicated time periods. In both sexes, no statistical difference was noted in baseline data of all cardiovascular data. In male dogs, a significant increase in QTcB and QTcF were observed at $4 \mathrm{~h}$ at $54 \mathrm{mg} / \mathrm{kg}$, at $3 \mathrm{~h}$ and $4 \mathrm{~h}$ at $540 \mathrm{mg} / \mathrm{kg}$, at $3 \mathrm{~h}$ and $4 \mathrm{~h}$ (QTcB only) at $1000 \mathrm{mg} / \mathrm{kg}$ as compared to vehicle control. These values, however, were compared to baseline data by paired $t$-test and no significant difference was noted except the QTcF value of $1000 \mathrm{mg} / \mathrm{kg}$ at $3 \mathrm{~h}$. These findings described above were not considered to be of toxicological significance because the changes were not dose related and within normal physiological range. Next, no statistical difference was noted in all cardiovascular data of female dogs (heart rate, RR interval, PR interval, $P$ duration, QRS duration, QT interval, and corrected QT (QTc)) at each indicated time period. Furthermore, the blood pressure parameters in $T$. camphoratus extract treated male and female dogs showed no statistical difference at each indicated time period as compared to vehicle control (Tables $4 \& 5$ ).

\begin{tabular}{|c|c|c|c|c|c|c|c|c|}
\hline Parameters & Gender & $\begin{array}{c}\text { Dose } \\
\mathbf{m g} / \mathbf{k g})\end{array}$ & Pre-dose & $\mathbf{1}$ h & $\mathbf{2} \mathbf{h}$ & $\mathbf{3 ~ h}$ & $\mathbf{4 h}$ & $\mathbf{2 4} \mathbf{h}$ \\
\hline RR Interval (ms) \\
\hline
\end{tabular}




\begin{tabular}{|c|c|c|c|c|c|c|c|c|}
\hline & Female & 0 & $93.587 \pm 6.351$ & $85.920 \pm 2.882$ & $88.937 \pm 8.371$ & $87.247 \pm 5.864$ & $87.880 \pm 8.315$ & $89.810 \pm 3.775$ \\
\hline & & 54 & $91.630 \pm 6.395$ & $89.163 \pm 3.995$ & $88.340 \pm 2.409$ & $85.183 \pm 3.445$ & $91.230 \pm 7.962$ & $91.010 \pm 5.254$ \\
\hline & & 540 & $90.647 \pm 7.097$ & $89.200 \pm 3.810$ & $90.050 \pm 8.087$ & $85.137 \pm 0.701$ & $88.273 \pm 4.077$ & $90.087 \pm 4.650$ \\
\hline & & 1000 & $99.893 \pm 12.184$ & $95.203 \pm 8.709$ & $94.670 \pm 11.690$ & $92.050 \pm 6.231$ & $90.963 \pm 8.570$ & $92.100 \pm 4.723$ \\
\hline \multicolumn{9}{|c|}{ P wave Duration (ms) } \\
\hline & Male & 0 & $35.770 \pm 5.350$ & $34.847 \pm 4.064$ & $35.080 \pm 4.052$ & $36.190 \pm 3.428$ & $42.330 \pm 4.476$ & $35.680 \pm 7.207$ \\
\hline & & 54 & $35.087 \pm 3.264$ & $35.843 \pm 3.360$ & $37.133 \pm 3.928$ & $37.340 \pm 4.688$ & $40.470 \pm 3.785$ & $36.537 \pm 4.505$ \\
\hline & & 540 & $39.120 \pm 4.793$ & $43.760 \pm 3.050$ & $37.997 \pm 5.213$ & $44.170 \pm 5.116$ & $40.577 \pm 3.131$ & $37.117 \pm 4.885$ \\
\hline & & 1000 & $36.080 \pm 4.402$ & $37.693 \pm 3.592$ & $37.277 \pm 3.406$ & $37.680 \pm 3.964$ & $36.847 \pm 4.052$ & $38.497 \pm 3.124$ \\
\hline & Female & 0 & $37.870 \pm 2.102$ & $36.037 \pm 1.409$ & $37.877 \pm 2.410$ & $36.637 \pm 2.249$ & $36.790 \pm 2.102$ & $38.213 \pm 2.027$ \\
\hline & & 54 & $37.043 \pm 6.300$ & $35.997 \pm 2.884$ & $35.347 \pm 4.119$ & $34.120 \pm 3.108$ & $38.560 \pm 8.982$ & $36.377 \pm 3.851$ \\
\hline & & 540 & $38.673 \pm 2.791$ & $36.793 \pm 2.010$ & $38.900 \pm 4.948$ & $35.290 \pm 2.266$ & $35.320 \pm 4.157$ & $35.713 \pm 3.203$ \\
\hline & & 1000 & $41.470 \pm 4.936$ & $39.493 \pm 4.003$ & $40.280 \pm 5.974$ & $39.307 \pm 4.157$ & $37.907 \pm 5.972$ & $37.160 \pm 2.833$ \\
\hline \multicolumn{9}{|c|}{ QRS wave Duration (ms) } \\
\hline & \begin{tabular}{|l|} 
Male \\
\end{tabular} & 0 & $40.737 \pm 3.340$ & $39.530 \pm 1.882$ & $39.440 \pm 1.384$ & $39.010 \pm 2.653$ & $38.933 \pm 3.622$ & $39.983 \pm 2.174$ \\
\hline & & 54 & $39.333 \pm 1.444$ & $39.197 \pm 2.113$ & $39.933 \pm 1.826$ & $39.103 \pm 1.985$ & $40.913 \pm 4.180$ & $39.880 \pm 1.228$ \\
\hline & & 540 & $39.610 \pm 3.879$ & $38.920 \pm 3.916$ & $39.927 \pm 3.435$ & $39.080 \pm 3.981$ & $39.963 \pm 3.591$ & $40.413 \pm 4.524$ \\
\hline & & 1000 & $38.860 \pm 2.223$ & $39.050 \pm 1.254$ & $39.387 \pm 1.030$ & $39.650 \pm 2.001$ & $39.280 \pm 1.508$ & $39.867 \pm 2.140$ \\
\hline & Female & 0 & $40.853 \pm 1.955$ & $38.577 \pm 2.916$ & $39.417 \pm 4.525$ & $38.740 \pm 3.248$ & $38.620 \pm 2.025$ & $42.017 \pm 6.946$ \\
\hline & & 54 & $40.373 \pm 4.674$ & $37.230 \pm 2.089$ & $38.600 \pm 3.212$ & $40.270 \pm 5.963$ & $37.597 \pm 2.752$ & $38.023 \pm 3.410$ \\
\hline & & 540 & $40.600 \pm 3.182$ & $38.343 \pm 2.361$ & $39.367 \pm 0.731$ & $38.863 \pm 2.486$ & $39.053 \pm 2.754$ & $0 \pm 1.573$ \\
\hline & & 1000 & $37.693 \pm 2.546$ & $37.073 \pm 1.848$ & $37.720 \pm 1.340$ & $37.073 \pm 1.409$ & $36.610 \pm 2.201$ & $37.757 \pm 2.214$ \\
\hline \multicolumn{9}{|c|}{ QT Interval (ms) } \\
\hline & Male & 0 & $206.057 \pm 1.141$ & $201.877 \pm 1.916$ & $206.367 \pm 5.905$ & $203.893 \pm 2.259$ & $202.930 \pm 3.292$ & $210.413 \pm 6.634$ \\
\hline & & 54 & $191.583 \pm 14.820$ & $186.527 \pm 11.450$ & $196.833 \pm 12.281$ & $195.250 \pm 9.689$ & $201.210 \pm 9.257$ & $202.630 \pm 5.552$ \\
\hline & & 540 & $204.273 \pm 20.341$ & $202.880 \pm 7.816$ & $200.253 \pm 12.000$ & $206.530 \pm 9.097$ & $197.860 \pm 10.753$ & $205.930 \pm 9.756$ \\
\hline & & 1000 & $201.030 \pm 12.067$ & $188.980 \pm 10.458$ & $195.600 \pm 8.354$ & $202.487 \pm 9.031$ & $196.073 \pm 4.124$ & $210.107 \pm 4.766$ \\
\hline & Female & 0 & $204.360 \pm 9.544$ & $190.747 \pm 6.205$ & $199.027 \pm 18.670$ & $197.817 \pm 10.616$ & $198.280 \pm 12.799$ & $203.600 \pm 19.481$ \\
\hline & & 54 & $195.887 \pm 12.476$ & $183.640 \pm 12.736$ & $191.120 \pm 8.444$ & $187.437 \pm 6.908$ & $198.163 \pm 12.725$ & $199.873 \pm 11.167$ \\
\hline & & 540 & $213.013 \pm 27.911$ & $198.193 \pm 20.540$ & $207.780 \pm 31.052$ & $196.570 \pm 8.557$ & $199.530 \pm 16.356$ & $209.890 \pm 20.110$ \\
\hline & & 1000 & $202.463 \pm 30.136$ & $197.110 \pm 26.206$ & $195.013 \pm 19.255$ & $201.963 \pm 29.364$ & $200.973 \pm 30.712$ & $200.737 \pm 20.575$ \\
\hline \multicolumn{9}{|l|}{ QTcB (ms) } \\
\hline & Male & 0 & $242.907 \pm 6.692$ & $243.660 \pm 6.197$ & $248.547 \pm 5.228$ & $241.397 \pm 9.210$ & $236.070 \pm 9.328$ & $258.943 \pm 15.129$ \\
\hline & & 54 & $258.383 \pm 8.989$ & $251.897 \pm 13.068$ & $252.303 \pm 12.607$ & $256.753 \pm 5.337$ & $263.070 \pm 7.214 \#$ & $242.643 \pm 6.930$ \\
\hline & & 540 & $254.887 \pm 1.825$ & $255.627 \pm 8.920$ & $257.947 \pm 7.875$ & $263.823 \pm 10.519 \#$ & $263.713 \pm 6.470 \mathrm{\#}$ & $240.467 \pm 10.800$ \\
\hline & & 1000 & $250.220 \pm 8.885$ & $256.427 \pm 12.486$ & $255.067 \pm 15.687$ & $260.670 \pm 4.626 \#$ & $260.097 \pm 8.056 \#$ & $259.977 \pm 16.944$ \\
\hline & Female & 0 & $256.907 \pm 19.104$ & $236.567 \pm 10.190$ & $249.990 \pm 20.813$ & $247.377 \pm 15.665$ & $245.403 \pm 14.907$ & $256.907 \pm 4.757$ \\
\hline & & 54 & $261.820 \pm 14.408$ & $254.100 \pm 10.237$ & $252.130 \pm 9.935$ & $249.173 \pm 10.426$ & $257.200 \pm 20.161$ & $256.577 \pm 12.554$ \\
\hline & & 540 & $259.277 \pm 32.079$ & $250.763 \pm 19.598$ & $253.230 \pm 27.836$ & $251.100 \pm 16.082$ & \begin{tabular}{|l}
$258.127 \pm 8.173$ \\
\end{tabular} & $252.877 \pm 9.634$ \\
\hline & & 1000 & $258.943 \pm 9.687$ & $257.490 \pm 5.664$ & $252.857 \pm 8.804$ & $254.310 \pm 16.623$ & $255.803 \pm 14.263$ & $253.970 \pm 17.136$ \\
\hline \multicolumn{9}{|c|}{ 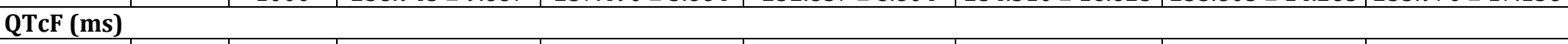 } \\
\hline & Male & 0 & $228.973 \pm 4.524$ & $228.053 \pm 4.147$ & $232.630 \pm 4.046$ & $227.137 \pm 5.765$ & $223.437 \pm 5.206$ & $240.823 \pm 9.602$ \\
\hline & & 54 & $233.347 \pm 7.490$ & $227.303 \pm 6.329$ & $231.440 \pm 5.177$ & $233.730 \pm 1.535$ & $239.993 \pm 3.820 \#$ & $227.633 \pm 3.101$ \\
\hline & & 540 & $235.830 \pm 6.452$ & $235.900 \pm 2.805$ & $236.320 \pm 2.015$ & $242.367 \pm 4.452 \#$ & $238.987 \pm 0.655$ \# & $227.510 \pm 3.836$ \\
\hline & & 1000 & $231.650 \pm 4.715$ & $231.150 \pm 4.893$ & $232.903 \pm 7.997$ & $239.080 \pm 4.492 *$ & $236.260 \pm 3.948$ \# & $241.460 \pm 9.898$ \\
\hline & Female & 0 & $237.030 \pm 14.782$ & $219.433 \pm 8.118$ & $230.733 \pm 18.505$ & $228.717 \pm 13.100$ & $227.553 \pm 12.429$ & $236.720 \pm 10.037$ \\
\hline & & 54 & $237.153 \pm 10.328$ & $227.547 \pm 10.176$ & $229.223 \pm 6.562$ & $226.117 \pm 6.983$ & $235.077 \pm 16.229$ & $235.297 \pm 10.258$ \\
\hline & & 540 & $241.640 \pm 26.874$ & $230.963 \pm 16.278$ & $236.070 \pm 28.170$ & $230.620 \pm 11.319$ & $236.103 \pm 9.233$ & $236.650 \pm 11.816$ \\
\hline & & 1000 & $237.367 \pm 12.446$ & $234.683 \pm 12.643$ & $231.050 \pm 6.433$ & $234.493 \pm 18.764$ & $235.080 \pm 18.502$ & $234.250 \pm 18.580$ \\
\hline \multicolumn{9}{|c|}{ Heart Rate (bpm) } \\
\hline & Male & 0 & $75.430 \pm 5.615$ & $80.237 \pm 3.134$ & $79.000 \pm 7.967$ & $75.750 \pm 8.963$ & $73.417 \pm 10.213$ & $84.697 \pm 11.660$ \\
\hline & & 54 & $106.190 \pm 19.256$ & $106.157 \pm 22.123$ & $93.740 \pm 21.483$ & $98.720 \pm 15.092$ & $97.927 \pm 14.099$ & $79.180 \pm 12.379$ \\
\hline & & 540 & $88.873 \pm 20.390$ & $89.490 \pm 14.260$ & $94.827 \pm 19.413$ & $92.583 \pm 17.472$ & $102.090 \pm 19.533$ & $76.803 \pm 14.618$ \\
\hline
\end{tabular}




\section{Advances in Clinical Toxicology}

\begin{tabular}{|l|c|c|c|c|c|c|c|c|}
\hline & & 1000 & $86.330 \pm 19.731$ & $108.670 \pm 24.613$ & $98.937 \pm 21.587$ & $94.643 \pm 10.827$ & $101.200 \pm 12.232$ & $86.973 \pm 15.244$ \\
\hline & Female & 0 & $85.930 \pm 11.608$ & $85.123 \pm 5.496$ & $87.067 \pm 15.555$ & $85.860 \pm 9.018$ & $83.920 \pm 12.151$ & $88.493 \pm 14.495$ \\
\hline & & 54 & $103.667 \pm 17.091$ & $110.410 \pm 14.069$ & $98.553 \pm 14.795$ & $101.367 \pm 10.892$ & $94.830 \pm 14.584$ & $92.300 \pm 12.997$ \\
\hline & & 540 & $83.207 \pm 23.738$ & $91.260 \pm 21.825$ & $82.417 \pm 12.844$ & $91.350 \pm 15.524$ & $94.813 \pm 16.372$ & $80.723 \pm 13.057$ \\
\hline & & 1000 & $96.020 \pm 35.797$ & $98.977 \pm 24.479$ & $97.157 \pm 25.056$ & $90.943 \pm 25.247$ & $93.757 \pm 24.830$ & $91.040 \pm 6.416$ \\
\hline
\end{tabular}

Table 4: Effect of T. camphoratus Extract on ECG Parameters in Beagle Dogs.

All data presented as mean \pm SD.

${ }^{*} p<0.05$ [Statistical difference from the control group by Dunnett's method and from baseline by paired $t$-test.]

$\# p<0.05$ [Statistical difference from the control group by Dunnett's method, but with no statistical difference from the baseline by paired $t$-test.].

\begin{tabular}{|c|c|c|c|c|c|c|c|c|}
\hline Parameters & Gender & $\begin{array}{c}\text { Dose } \\
(\mathrm{mg} / \mathrm{kg})\end{array}$ & Pre-dose & $1 \mathrm{~h}$ & $2 \mathrm{~h}$ & $3 \mathbf{h}$ & $4 \mathrm{~h}$ & $24 \mathrm{~h}$ \\
\hline \multicolumn{9}{|c|}{ Diastolic Arterial Pressure (mmHg) } \\
\hline & Male & 0 & $71.723 \pm 10.717$ & $74.730 \pm 5.697$ & $72.787 \pm 17.095$ & $85.827 \pm 22.943$ & $71.780 \pm 9.820$ & $72.033 \pm 8.915$ \\
\hline & & 54 & $75.653 \pm 13.462$ & $84.387 \pm 31.292$ & $64.973 \pm 13.569$ & $72.243 \pm 8.954$ & $72.173 \pm 16.368$ & $73.593 \pm 6.770$ \\
\hline & & 540 & $70.243 \pm 6.934$ & $67.113 \pm 5.577$ & $65.300 \pm 10.888$ & $67.040 \pm 2.746$ & $75.883 \pm 10.167$ & $72.167 \pm 4.491$ \\
\hline & & 1000 & $64.037 \pm 8.878$ & $82.787 \pm 33.932$ & $72.613 \pm 27.037$ & $62.340 \pm 13.232$ & $54.913 \pm 8.358$ & $64.083 \pm 7.796$ \\
\hline & Female & 0 & $67.503 \pm 12.804$ & $82.767 \pm 10.919$ & $74.023 \pm 4.972$ & $68.727 \pm 5.416$ & $67.130 \pm 9.918$ & $70.747 \pm 14.513$ \\
\hline & & 54 & $61.410 \pm 5.577$ & $70.997 \pm 9.352$ & $61.027 \pm 9.095$ & $68.193 \pm 5.443$ & $81.777 \pm 26.263$ & $64.497 \pm 6.579$ \\
\hline & & 540 & $64.857 \pm 4.945$ & $66.803 \pm 1.589$ & $65.270 \pm 7.665^{a}$ & $76.647 \pm 15.126$ & $61.960 \pm 8.825$ & $63.763 \pm 4.128$ \\
\hline & & 1000 & $64.527 \pm 8.057$ & $71.050 \pm 8.440$ & $65.943 \pm 6.659$ & $56.537 \pm 10.009$ & $60.953 \pm 7.543$ & $59.430 \pm 15.599 \mathrm{a}$ \\
\hline \multicolumn{9}{|c|}{ Systolic Arterial Pressure (mmHg) } \\
\hline & Male & 0 & $119.173 \pm 19.173$ & $129.633 \pm 15.506$ & $123.827 \pm 28.079$ & $132.487 \pm 21.398$ & $116.603 \pm 7.987$ & $119.427 \pm 18.234$ \\
\hline & & 54 & $122.290 \pm 15.031$ & $140.467 \pm 30.572$ & $126.777 \pm 21.013$ & $123.560 \pm 10.139$ & $123.687 \pm 15.826$ & 11.099 \\
\hline & & 540 & $126.227 \pm 16.320$ & $121.060 \pm 5.983$ & $120.343 \pm 17.277$ & $121.477 \pm 15.623$ & $125.267 \pm 12.978$ & $127.173 \pm 19.665$ \\
\hline & & 1000 & $108.950 \pm 6.190$ & $123.440 \pm 24.995$ & $118.163 \pm 18.314$ & $104.717 \pm 7.843$ & $109.430 \pm 10.546$ & $6115.063 \pm 16.114$ \\
\hline & Female & 0 & $106.443 \pm 10.599$ & $124.920 \pm 11.499$ & $121.900 \pm 16.123$ & $116.410 \pm 13.126$ & $120.020 \pm 22.471$ & $116.490 \pm 10.157$ \\
\hline & & 54 & $105.440 \pm 5.856$ & $116.863 \pm 12.171$ & $105.197 \pm 6.617$ & $110.187 \pm 4.226$ & $128.307 \pm 32.081$ & $109.897 \pm 11.591$ \\
\hline & & 540 & $104.160 \pm 18.689$ & $115.327 \pm 9.046$ & $111.595 \pm 9.595 \mathrm{a}$ & $115.917 \pm 11.057$ & $102.357 \pm 5.889$ & $101.993 \pm 9.488$ \\
\hline & & 1000 & $103.693 \pm 5.809$ & $111.997 \pm 11.882$ & $110.540 \pm 12.109$ & $103.937 \pm 8.532$ & $103.387 \pm 6.739$ & $106.865 \pm 9.991$ a \\
\hline \multicolumn{9}{|c|}{ Mean Arterial Pressure (mmHg) } \\
\hline & Male & 0 & $93.233 \pm 14.943$ & $99.557 \pm 11.079$ & $97.340 \pm 25.772$ & $106.733 \pm 23.823$ & $91.627 \pm 9.782$ & $93.693 \pm 14.800$ \\
\hline & & 54 & $96.060 \pm 11.596$ & $111.737 \pm 31.787$ & $90.910 \pm 9.945$ & $93.963 \pm 10.440$ & $94.610 \pm 16.973$ & $97.237 \pm 7.714$ \\
\hline & & 540 & $97.303 \pm 11.184$ & $94.377 \pm 6.963$ & $88.163 \pm 14.131$ & $93.830 \pm 12.647$ & $98.510 \pm 11.621$ & $99.267 \pm 17.554$ \\
\hline & & 1000 & $86.113 \pm 5.078$ & $100.857 \pm 30.118$ & $92.763 \pm 23.177$ & $81.473 \pm 10.426$ & $73.910 \pm 8.958$ & $84.627 \pm 10.630$ \\
\hline & Female & 0 & $84.833 \pm 12.458$ & $100.740 \pm 9.972$ & $95.927 \pm 9.899$ & $89.720 \pm 7.922$ & $88.547 \pm 13.674$ & $90.990 \pm 10.507$ \\
\hline & & 54 & $81.087 \pm 6.153$ & $91.720 \pm 10.414$ & $81.060 \pm 7.453$ & $88.200 \pm 5.587$ & $101.930 \pm 27.703$ & $85.550 \pm 9.611$ \\
\hline & & 540 & $82.320 \pm 10.849$ & $88.760 \pm 3.631$ & $87.025 \pm 8.224^{a}$ & $95.253 \pm 14.125$ & $79.360 \pm 5.323$ & $80.507 \pm 8.033$ \\
\hline & & 1000 & $82.310 \pm 5.916$ & $88.903 \pm 10.707$ & $85.977 \pm 9.414$ & $77.630 \pm 9.768$ & $80.873 \pm 6.403$ & $76.955 \pm 17.458 \mathrm{a}$ \\
\hline
\end{tabular}

Table 5: Effect of T. camphoratus Extract on Blood Pressure in Beagle Dogs.

All data presented as mean \pm SD.

$\mathrm{a}: \mathrm{N}=2$ (The peak blood pressure of one female at $540 \mathrm{mg} / \mathrm{kg}$ was unrecognizable during 1 to $2 \mathrm{~h}$ post-dosing period and cannot be calculated reliably by iox system. In addition, no blood pressure was recorded in one female at $1000 \mathrm{mg} / \mathrm{kg}$ during 23 to $24 \mathrm{~h}$ post-dosing period, because the tail cuff was loose. Therefore, the data were not included in statistical calculation.)

The ECG morphology at each indicated time period was evaluated by a veterinary cardiologist that the sinus arrest noted in all six dogs and large negative $\mathrm{T}$ wave

noted in two dogs before and after the T. camphoratus extract treatment (data not shown). In general, $T$ wave changes are very non-specific. Tall $\mathrm{T}$ wave could be as a 


\section{Advances in Clinical Toxicology}

normal variant. This could occur with hyperventilation, anxiety, and even positional changes. However, tall $\mathrm{T}$ wave could also be a warming sign of myocardial hypoxia or electrolytes disturbance (hyperkalemia). Sinus arrest is frequently caused by high parasympathetic tone due to one or many factors. It is commonly in brachycephalic breed dogs with strenuous respiratory efforts that irritate the pharynx and cause reflex vagal stimulation. Other factors may cause sinus arrest include surgical stimulation, impingement upon the vagus nerve (neoplasia), drug toxicity (digitalis or $\beta$-blockers). No treatment is needed for this conducting disturbance, unless syncope is developed [18,19]. In this study, large (negative) $\mathrm{T}$ wave was found in one male and one female dog and sinus arrest was seen in all six dogs in both the predose and post stages. The link between these abnormal findings and T. camphoratus extract was not indicated. Based on the results, the dogs received $T$. camphoratus extract via oral administration up to the dosage of $1000 \mathrm{mg} / \mathrm{kg}$ did not cause physiological abnormalities on cardiovascular system in this study.

\section{Conclusion}

Results from in vitro hERG test and in vivo core batteries of safety pharmacology studies revealed that $T$. camphoratus extract had no significant effect on the hERG current at nominal concentrations of up to $25 \mu \mathrm{g} / \mathrm{mL}$ and had no obvious toxicity evidences on central nervous, respiratory and cardiovascular system (up to doses at $1700 \mathrm{mg} / \mathrm{kg}$ in female rats, $3400 \mathrm{mg} / \mathrm{kg}$ in male rats and $1000 \mathrm{mg} / \mathrm{kg}$ in both sexes of dogs). The results would provide the evidences to support the safety of $T$. camphoratus extract as a food supplement and for clinical usage.

\section{Conflict of Interests}

The authors declare that there are no conflicts of interests.

\section{References}

1. Geethangili M, Tzeng YM (2011) Review of Pharmacological Effects of Antrodia camphorata and Its Bioactive Compounds. Evid Based Complement Alternat Med 2011: 17.

2. Lu MC, El-Shazly M, Wu TY, Du YC, Chang TT, et al. (2013) Recent research and development of Antrodia cinnamomea. Pharmacol Ther 139(2): 124-156.
3. Wang JJ, Wu CC, Lee CL, Hsieh SL, Chen JB, et al. (2017) Antimelanogenic, Antioxidant and Antiproliferative Effects of Antrodia camphorata Fruiting Bodies on B16-F0 Melanoma Cells. PLoS One 12(1): e0170924.

4. Wu MD, Cheng MJ, Wang WY, Huang HC, Yuan GF, et al. (2011) Antioxidant activities of extracts and metabolites isolated from the fungus Antrodia cinnamomea. Nat Prod Res 25(16): 1488-1496.

5. Lu MC, Du YC, Chuu JJ, Hwang SL, Hsieh PC, et al. (2009) Active extracts of wild fruiting bodies of Antrodia camphorata (EEAC) induce leukemia HL 60 cells apoptosis partially through histone hypoacetylation and synergistically promote anticancer effect of trichostatin A. Arch Toxicol 83(2): 121-129.

6. Yen IC, Lee SY, Lin KT, Lai FY, Kuo MT, et al. (2017) In Vitro Anticancer Activity and Structural Characterization of Ubiquinones from Antrodia cinnamomea Mycelium. Molecules 22(5).

7. Chiu HW, Hua KF (2016) Hepatoprotective Effect of Wheat-Based Solid-State Fermented Antrodia cinnamomea in Carbon Tetrachloride-Induced Liver Injury in Rat. PLoS One 11(4): e0153087.

8. Li ZW, Kuang Y, Tang SN, Li K, Huang Y, et al. (2017) Hepatoprotective activities of Antrodia camphorata and its triterpenoid compounds against CCl4-induced liver injury in mice. J Ethnopharmacol 206: 31-39.

9. Huang TT, Wu SP, Chong KY, Ojcius DM, Ko YF, et al. (2014) The medicinal fungus Antrodia cinnamomea suppresses inflammation by inhibiting the NLRP3 inflammasome. J Ethnopharmacol 155(1): 154-164.

10. Chen YY, Lo CP, Lin CC, Hsieh YH (2018) Effects of Taiwanofungus camphoratus on non-specific and specific immune activities in mice. Mycology 9(2): 129-135.

11. Lin YH, Kuo JT, Chen YY, Kumar KJS, Lo CP, et al. (2018) Immunomodulatory Effects of the Stout Camphor Medicinal Mushroom, Taiwanofungus camphoratus (Agaricomycetes)-Based Health Food Product in Mice. Int J Med Mushrooms 20(9): 849858.

12. Yue PY, Wong YY, Chan TY, Law CK, Tsoi YK, et al. (2012) Review of biological and pharmacological activities of the endemic Taiwanese bitter medicinal 
mushroom, Antrodia camphorata (M Zang et $\mathrm{CH} \mathrm{Su}$ ) $\mathrm{Sh}$. H. Wu et al. (higher Basidiomycetes). Int J Med Mushrooms 14(3): 241-256.

13. Chang JB, Wu MF, Lu HF, Chou J, Au MK, et al. (2013) Toxicological evaluation of Antrodia cinnamomea in BALB/c mice. In Vivo 27(6): 739-745.

14. Huang CC, Nam MK, Tsai YT, Lan A (2014) Evaluation of the Sub-Chronic Toxicity and Teratogenicity of Antrodia cinnamomea mycelia. Life Sci J 11(11): 1090-1098.

15. Lin YH, Kumar KJS, Vani MG, Liao MG, Lin CC, et al. (2016) In vitro and in vivo toxicological assessments of Antrodia cinnamomea health food product (Leader Antrodia cinnamomea Capsule). Fundamental Toxicological Sciences 3(5): 205-216.

16. Lin CC, Kumar KJS, Liao JW, Kuo YH, Wang SY, et al. (2015) Genotoxic, teratotoxic and oral toxic assessments of Antrodia cinnamomea health food product (Leader Deluxe Antrodia cinnamomea (R)). Toxicol Rep 2: 1409-1417.

17. Lo CP, Chen YY, Lin CC, Kumar KJS (2016) Genotoxicity, Acute and Sub-Chronic Toxicity Studies of Solid-State Cultivated Mycelial Powder of Antrodia cinnamomea. Advances in Clinical Toxicology 1(1): 110.

18. Euler DE, Guo H, Olshansky B (1996) Sympathetic influences on electrical and mechanical alternans in the canine heart. Cardiovasc Res 32(5): 854-860.

19. Fox PR, Sisson D, Moïse NS (1999) Textbook of canine and feline cardiology: principles and clinical practice. $2^{\text {nd }}$ (Edn.), Philadelphia; London: Saunders. 10(6): 955. 